\title{
Planet Discoverer Interferometer (PDI) I: a potential precursor to Terrestrial Planet Finder
}

Neville J. Woolf, James Roger P. Angel, Charles A. Beichman, James H. Burge, Michael Shao, et al.

Neville J. Woolf, James Roger P. Angel, Charles A. Beichman, James H. Burge, Michael Shao, Domenick J. Tenerelli, "Planet Discoverer Interferometer (PDI) I: a potential precursor to Terrestrial Planet Finder," Proc. SPIE 3350, Astronomical Interferometry, (24 July 1998); doi: 10.1117/12.317132 


\title{
Planet Discoverer Interferometer I: PDI, a potential precursor to Terrestrial Planet Finder
}

\author{
N.J. Woolf ${ }^{\text {a }}$, J.R.P. Angel ${ }^{\text {a }}$, C.A. Beichman ${ }^{\text {b }}$, J. Burge ${ }^{\text {a }}$, M. Shao ${ }^{c}$ and D. Tenerelli ${ }^{d}$ \\ ${ }^{a}$ Steward Observatory, University of Arizona, Tucson AZ \\ ' IPAC, California Institute of Technology, Pasadeńa CA \\ 'Jet Propulsion Laboratory, Pasadena CA \\ ${ }^{\mathrm{d}}$ Lockheed Martin, Missiles and Space, Mountain View CA
}

\begin{abstract}
We consider a possible precursor interferometer to Terrestrial Planet Finder. The precursor called Planet Discoverer Interferometer (PDI) would search for broadband $10 \mu \mathrm{m}$ radiation from possible terrestrial planets orbiting stars out to a distance of $8-10 \mathrm{pc}$ and at an angular separation of at least 0.1 arcseconds. There are about 20 stars of types A,F,G and K around which an Earth-analog might be detected. PDI would be able to confirm such planets by seeing their orbital motion. PDI would also be able to observe $5 \mu \mathrm{m}$ radiation from the more massive and younger gas-giant planets around stars up to distances $\sim 150 \mathrm{pc}$, separated from their star by more than 0.05 arc seconds. It would also see the re-radiated thermal radiation of Jupiter-like planets at temperatures above $\sim 130 \mathrm{~K}$. The device would be a $15 \mathrm{~m}$ long truss with four SIRTF-like telescopes. It would need to be in a SIRTF-like Earth-trailing orbit, and would be radiatively cooled. A very preliminary design suggests that PDI could fit into the shroud of a Delta II rocket. Similar preliminary calculations suggest that the total lifetime cost of such a mission would be under $\$ 300 \mathrm{M}$. Detailed studies of this concept are in process.
\end{abstract}

Keywords: Extrasolar , terrestrial, planets, interferometer, Origins.

\section{INTRODUCTION}

The Origins Program, a major NASA scientific, was developed to address the fundamental question of whether life is common in the universe ---a question of interest to both scientists and the person in the street. Terrestrial Planet Finder was conceived as a way to find the arrangement of planets in nearby planetary systems, and to determine whether the atmospheres of earth-like planets in those systems show evidence of life. The issue of this paper is to explore whether a direct precursor to TPF might minimize cost and risk, while maximizing the speed of both return of important scientific results, and technological developments needed for subsequent steps of the program. The work in this paper is a blend of work before the Kona meeting, and conclusions already accepted by the TPF Science Working Group (TPF-SWG) with new work initiated at the Kona meeting.

\section{THE "OLD" TPF}

The TPF concept originated in 1995 with the JPL Ex-NPS study, the Exploration of Nearby Planetary Systems ${ }^{1}$. It built on earlier studies ${ }^{2,3}$. These earlier studies concluded first that the task of observing the spectra of Earth-like planets required the "Sun" of the system to be blotted out by a broad, deep interferometric null that was better than could be produced by a two-beam interferometer. Secondly that such an interferometer is used at $1 \mathrm{AU}$ from the Sun would have its noise set by the IR emission from solar system dust and its photon fluctuations. And in consequence each interferometer mirror would need to be larger than the Hubble Space Telescope.

Leger et al. proposed therefore that an interferometer with much smaller mirrors e.g. 1 meter diameter could be adequate if it were operated at 3-5 AU from the Sun, where the dust was much colder, and its thermal emission in the $10 \mu \mathrm{m}$ region correspondingly reduced. This became a part of the initial baseline concept ${ }^{1,4}$. 


\section{MODIFYING THE CONCEPT}

Careful examination of noise sources which interfere with the detection of planets, highlighted the importance of dust emission. And though the emission of Solar System dust is crudely known, the $\sim 10 \mu$ emission of other systems is currently unknown and unpredictable 5 . A program was proposed to make the necessary measurements from the ground ${ }^{6}$ using the Large Binocular telescope, and was also proposed for the Keck Interferometer.

If other planetary systems have more dust emission from the $1 \mathrm{AU}$ range than does our solar system, then an interferometer at $5 \mathrm{AU}$ would require greatly increased observing time, or increase of the telescope size. And indeed, if the planetary system dust were much brighter than solar system dust emission at $1 \mathrm{AU}$, the same size mirrors would be needed whether TPF were at $1 \mathrm{AU}$ or $5 \mathrm{AU}$. TPF-SWG considered possible amounts of bright dust that would still leave planet detectable, and decided that interferometer mirrors at $5 \mathrm{AU}$ would need to be $\sim 2 \mathrm{~m}$ diameter each, whereas at $1 \mathrm{AU}$ similar signal/noise ratio would be achieved with $\sim 4.5 \mathrm{~m}$ mirrors.

The major event since the release of the Ex-NPS report is the dramatic development of low-cost lightweight optics for NGST. Steward Observatory Mirror Lab has modified technology that was being developed to make adaptive optics secondaries for the MMT conversion and for the Large Binocular Telescope, and now expects to make large lightweight telescope primaries ${ }^{7}$. Other industrial groups are pursuing different but equally promising technologies for lightweight optics. NGST is paying for their development. Four mirrors each of half the area of NGST now seem practicable for TPF.

The TPF Science Working Group has decided to change the baseline TPF mission to be at 1AU because there are also a number of other accompanying benefits. First, the science results would come in a more useful form. A 1 AU fall-away Earth-trailing orbit like SIRTF's would allow the entire sky to be surveyed in one year. A complete survey for planets could be done in that time, with the follow up spectroscopic observations being planned for the following year, rather than being a hurried response to what had just been observed. There will no longer be a 10year wait for the interferometer to encounter that part of the sky again. There will no longer be a long wait after launch while the interferometer travels to its operating location. Also a number of technical problems are eased. IR detectors do not need to be so noise free, because they will now work in a stronger radiation environment. There is no longer a need for giant solar cells or expensive communication links. The interferometer is no longer so sensitive to the exact amount of zodiacal dust (though it still helps designs to know how much to expect.) The complexity of operating a deep-space observatory is eliminated.

\section{PRECURSORS}

No spatial interferometer has yet flown. How should we best get from this situation to successfully flying a large, complex and novel observatory?

The path laid out in Origins planning envisioned two interferometric precursors to TPF. One of them is DS3, an experiment to explore the problems of using separated spacecraft as an interferometer. The other is SIM. Both were discussed at this meeting. Both would be devices only used at visible wavelengths. SIM would include a nulling experiment that would demonstrate path length control at visible wavelengths adequate for TPF.

Additional precursor options have been explored. The simplest interferometer would be a two-element Bracewell interferometer that would null the starlight to reveal the zodiacal emission of the external system. Bely ${ }^{8}$ at this meeting, proposed an example of such a device, to be used at $1 \mathrm{AU}$. But as has been already pointed out, this measurement can be made from the ground at much less expense. There still remains the question of whether some other kind of precursor would so reduce the risk and cost of TPF as to make a precursor mission a cost effective part of the TPF program. Alternately, a Discovery or Midex class mission might be justified on the basis of the science alone, but with the added advantage of advancing the technology.

Three industrial teams from Ball, Lockheed-Martin and TRW recently studied aspects of TPF, and the question of whether there should be a precursor mission was posed to them. The three teams agreed with each other on two somewhat contradictory statements. The first was that money would better be spent on ground experiments to ensure the success of the TPF mission. But the second was that the cost of TPF would not be so much for the components that flew as for the supporting activity that could convince non-advocates (not to mention the advocates!) that the mission would be a success. It is hard to imagine any more convincing evidence than a successful mission on a smaller scale. And on a smaller scale, ground based testing of the flight equipment is also possible. 


\section{THE KONA ACTIVITY}

As part of the discussion a this meeting, a number of us agreed that while the study of exozodiacal dust represented an inadequate science goal for a dedicated mission, the detection of Earth-like planets even without spectroscopic observations might represent a worthwhile science mission. Such a device could also detect the thermal emission of a number of giant planets. PDI (or Paddy) was born.

A number of technical considerations led to the idea that a mission of modest scope could provide significant scientific return.

1. SIRTF optics represent a developed technology at a fixed cost. A PDI using four "built to print" SIRTF mirrors would eliminate the need for optics development.

2. SIRTF's $10 \mu \mathrm{m}$ detectors are well characterized low risk devices.

3. The SIRTF spacecraft is a known quantity that would be adequate for PDI. Even though PDI has a much larger moment of inertia than SIRTF, it would always be slewed very slowly.

4. Since PDI would operate only at wavelengths shorter than $20 \mu \mathrm{m}$, and with a small heat load, no expensive, massive liquid helium cryostat would be needed. A simple solid hydrogen cryostat such as has been developed for WIRE would be suffice. A number of low power coolers are close to space qualification for $<10 \mathrm{~K}$ operation. These would eliminate the need for a heavy cryogenic vacuum vessel and remove a $\sim 2$ year lifetime restriction of PDI operations.

5. No large scale delay lines would be needed. Since PDI would always work "on-axis", the delay lines for PDI would only need a few $\mathrm{cm}$ of travel. The medium and fine delay lines developed for SIM could be adapted for cryogenic operation on PDI with well understood flex-pivot and PZT designs already qualified for ISO and SIRTF.

6. The speedy improvement in nulling would be a principal development activity needed for PDI. However, we have advanced from it merely being a theoretical concept. Nulling is now a real observational tool, having been both demonstrated in the laboratory by Serabyn (to be published), and at the telescope as reported ${ }^{9}$ at this meeting.

\section{THE OPTICAL CONFIGURATION}

The interferometer configuration was developed after considering a number of possible designs.

A 10m long 2-telescope Bracewell nulling interferometer could resolve planets at $1 \mathrm{AU}$ from their star out to 10pc distance. But such a device would be unable to distinguish a planet signal from the signal of an inclined exo-zodiacal dust disk because there would be two signals from the planet and two from the dust for each interferometer revolution.

If the device were expanded to $20 \mathrm{~m}$ to give the planet 4 signals per revolution, the null would shrink so much that starlight leakage photon noise would prevent planet detection.

The null could be made broader by using a 3 or 4 element interferometer. This would a variant of the 1-2-1 configuration ${ }^{10}$. But the length of the interferometer would now expand to $40 \mathrm{~m}$.

We can make two $10 \mathrm{~m}$ long devices as in item 1 above. We can interlace them so that the four telescopes have $5 \mathrm{~m}$ gaps between them, and the device is $15 \mathrm{~m}$ long overall. We can combine the signals from the two nulled outputs with a $1 / 4$ wave shift between them. Such a device gives the same angular resolution as in item 1. It would also separate the planet from the star by giving asymmetric response. See figure 1 . It would have a just-wide-enough null for the task. This concept of double interferometers was proposed by Shao and developed by Woolf and Angel ${ }^{10}$. We propose using this configuration using four SIRTF-like $85 \mathrm{~cm}$ telescopes.

\section{THE PDI OPTICS ARE TECHNICALLY SIMPLER THAN TPF}

We explored the similarities and differences of the nulling needs for PDI and TPF. PDI can get by with a lower quality null than TPF. The solid angle seen by the detectors of both systems would collect the same size background from solar system zodiacal dust. However, the telescope collecting area of TPF would be 22 times larger than for PDI. As a result a lower quality null would still leave PDI limited by the zodiacal dust radiation. Further, PDI offers a chopping solution that can substantially reduce the effects of some starlight leaking onto the detectors, and with that leak varying with time.

The chopping scheme of the PDI concept is directly applicable to TPF also. And the compactness of PDI can also be carried over somewhat to TPF, with $50 \mathrm{~m}$ versions of TPF performing at a level similar to $75-100 \mathrm{~m}$ versions previously considered. 


\section{SPATIAL RESPONSE FROM THE OPTICAL CONFIGURATION OF SECTION 6}
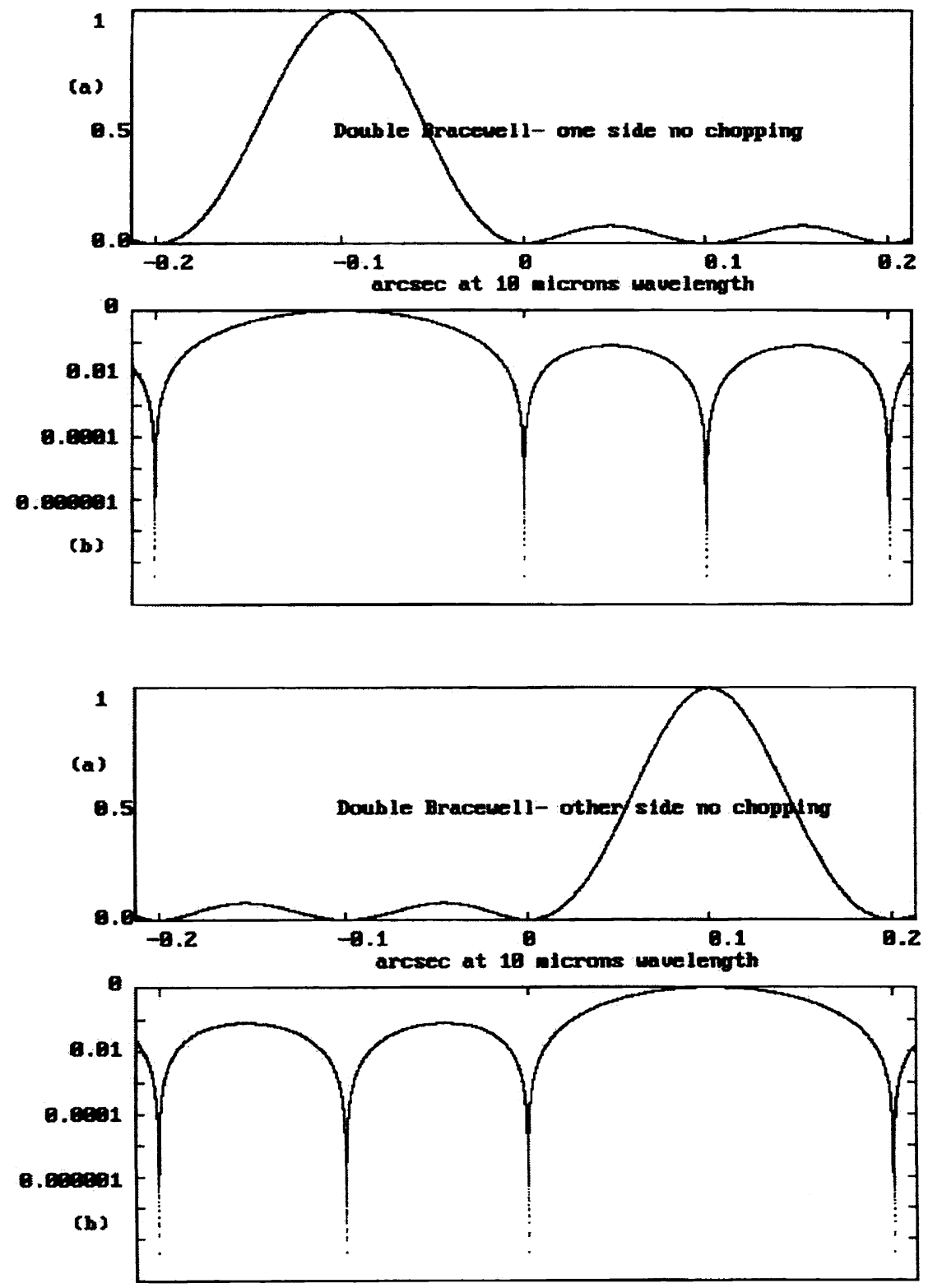

Figure 1. Transmission at $10 \mu \mathrm{m}$ of a $15 \mathrm{~m}$ long interferometer. Four telescopes are spaced with $5 \mathrm{~m}$ gaps between. The two interferometer outputs are shown on linear and logarithmic scales.

Each output would be led to its own detector, but chopping by phase adjustment would alternately let each detector see one or the other output. Note that the signals distinguish between planets on the right and left, and that a symmetric dust cloud about the star would produce no chopped signal (though its signal could be recovered). 


\section{FITTING INTO A DELTA II SHROUD}

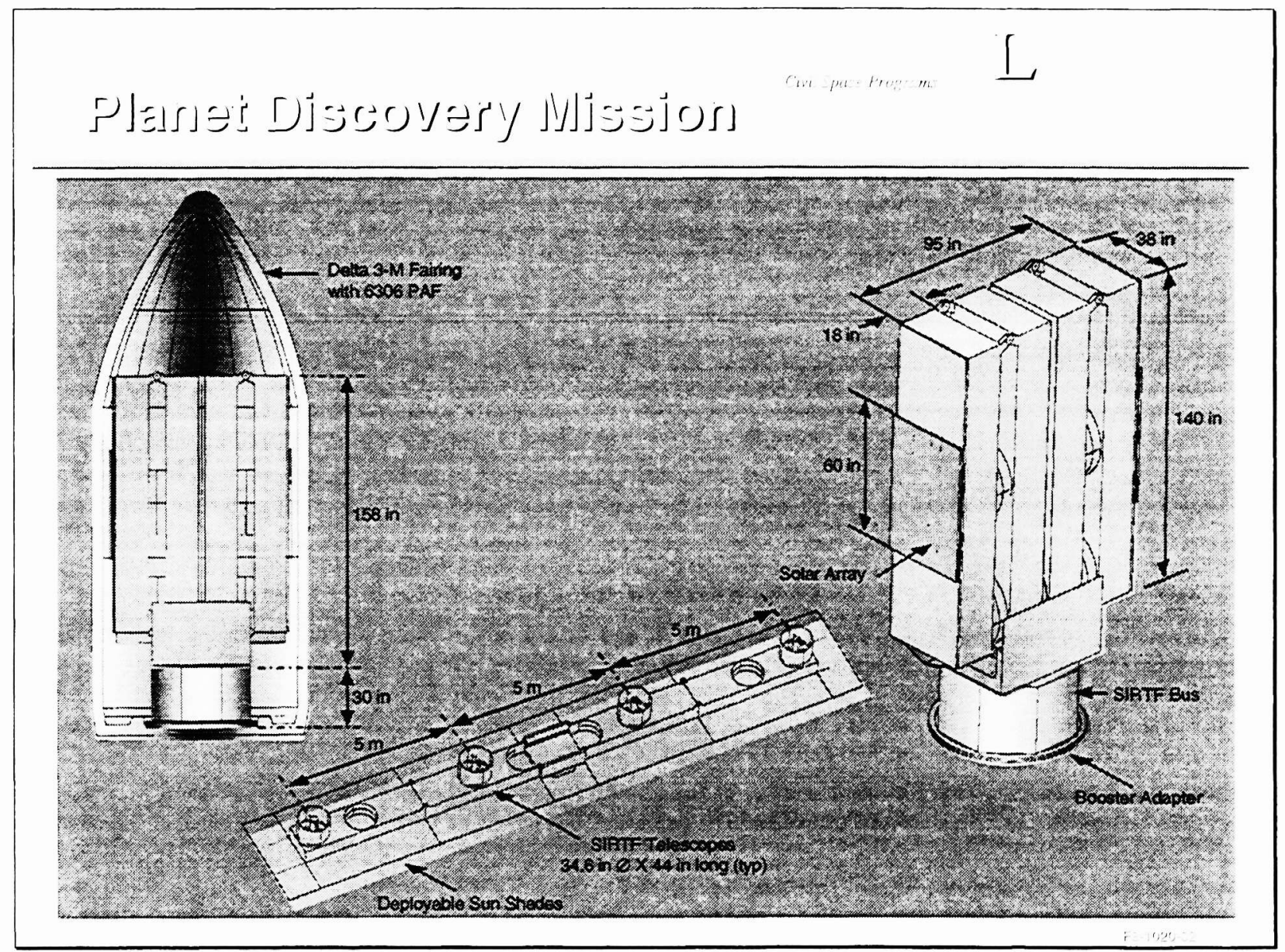

Figure 2. Shows a highly preliminary sketch of how such a device might be folded and fitted into the shroud of a Delta II rocket capable of carrying it into a 1 AU fall-away orbit like SIRTF.

This is a simple proof-of-concept diagram. The thermal shield would need to be isolated from the truss. The SIRTF bus would need to have an anti-vibration mounting to couple it to the truss, as is being developed for SIM. The concept of embedding the telescope optics in the truss is useful for folding the truss, but implies that the optical links between the telescopes are carried along the sides of the truss. To keep the truss strong, the telescopes would not have shrouds. Adequate shielding could be provided in the column that carried the secondary, and that could be arranged so that it fitted hetween truss members. The solar panels would probably need to be separately attached to the bus.

\section{ANALYSIS OF SENSITIVITY}

We start by comparing the sensitivity of PDI to a TPF with similar detector performance and optical efficiency. Then we try an absolute calculation

Because the total collecting area of TPF would be 22 times greater than PDI, but both would see the same zodiacal flux , and so for the same observation in the same time TPF would give 22 times as great a signal/noise ratio, if the optical efficiency is the same for both devices. Let us imagine we need a spectroscopic $\mathrm{S} / \mathrm{N}$ of 20 on an earth-like planet with TPF and that it has a bandpass of 0.5 micron, and needs to look at objects twice as far away as PDI. Let PDI observe with a bandwidth of 4 microns and require a $\mathrm{S} / \mathrm{N}$ of 5 . Then one finds that the observing time for one planetary system with TPF 
needs to be 4 times longer than with PDI. Since limiting observation times with TPF have been projected as $\sim 2$ months, this would make the necessary time per planetary system with PDI two weeks.

The observing time has also been calculated directly by sing the numbers from Angel and Woolf's study ${ }^{11}$ of the sensitivity of an active space telescope to faint sources and extrasolar planets. Let us assume the detector quantum efficiency is 0.3 , the transmission of the zodiacal signal is $50 \%$ because of optical inefficiency, and that some $30 \%$ of planet and star signals are rejected by the lambda squared throughput. PDI collecting area is 2.2 square meters. Each detector will observe the planet peak for $1 / 4$ of the observing time because the mean of the $\sin ^{2}$ transmission is $50 \%$, and we will be comparing planet with background, both suffering from zodical emission photon noise.

We find the zodiacal flux at $10 \mathrm{mu}=10 \mathrm{MJy}$. In a throughput of $10^{-10} \mathrm{~m}^{2}$ ster there will be $1000 \mu \mathrm{Jy}$, and this corresponds to 1000 counts per second in a bandwidth of 4 microns with an efficiency of 0.15 . The star will be at $8 \mathrm{pc}$ and its flux will be $3.5 \mathrm{Jy}$ and the planet flux $0.3 \mu \mathrm{Jy}$, so that in the 2.2 square meter aperture overall, 4 micron bandpass, and with overall efficiency 0.1 , the counts per second will be from the planet 0.5 , and from the star $5.4 \times 10^{6}$.

For the zodiacal flux to dominate, the star signal must be reduced by at least a factor $10^{4}$ by nulling. The planet signal will be observed on average $1 / 4$ of the time for each detector, and will be measured against a noise level set by the zodiacal signal. Thus in each second the planet signal/noise will be 0.007 . To bring this up to a S/N of 5 will take $5 \times 10^{5}$ seconds or 6 days. This observing time is acceptable, and within a factor 2 of the time suggested in the TPF comparison above. The nulling required is within the range of single beam-splitter techniques. Long term average phase stability is not changed from TPF, but the short term fluctuations can be about 10 times greater, and fall within the range already demonstrated by SIM studies.

\section{SCIENCE WITH PDI}

Analysis of sensitivity reveals that PDI could detect signals of less than $1 \mu \mathrm{Jy}$. That sensitivity coupled with the ability to separate the planet from a star 0.1 arcsecond away makes PDI appropriate for looking for Earth-like planets around some 20 or so A, F, G and $\mathrm{K}$ stars at distances up to 8-10pc. For the lowest luminosity stars, the angular resolution becomes the limit, and for higher luminosity stars, it is the faintness of an Earth-like planet at large distances. There are a further halfdozen or so stars at somewhat greater distances where spectroscopic searches have already revealed the presence of planets. Some of these are expected to have Jupiter-like planets that would be detectable by PDI, and all are worth searching for Earth-temperature planets. In a year of observation there would be the possibility of putting in 2 weeks of observation on each one of these -26 targets. Those that revealed the presence of the most interesting signals could then be re-examined for evidence of orbital motion during the following year.

Currently most astronomers consider that there is a high probability of planets forming around a single star. The observations discussed above would reveal whether that was correct. If it were not correct, and earth-like planets were far rarer than that, then PDI would easily determine that. It would substantially change the plans for TPF. On the other hand, if a number of bright planets were found in the nearby sample, then it would be important to plan TPF so that it could study these close-by objects in as great a detail as their flux permitted.

One part of the PDI second year study would be that the $10 \mu \mathrm{m}$ region would be observed in three spectral bands. So the comparison of the flux in the 7-9 $\mu \mathrm{m}$ region with that in 9-11 and 11-13 would reveal whether there was a precipitous drop such as would be caused by the presence of atmospheric gases $\mathrm{H}_{2} \mathrm{O}$ or $\mathrm{CH}_{4}$ in large quantities. Those would be particularly indicative of an object worthy of detailed study.

PDI would be a powerful tool for observing gas giant planets as well as terrestrial planets. Gas giant planets tend to put out about double the signal in the $5 \mu \mathrm{m}$ window that they do at $10 \mu \mathrm{m}$. And a $5 \mu \mathrm{m}$ detector will not need cooling beyond the $\sim 30 \mathrm{~K}$ to $40 \mathrm{~K}$ that PDI can reach passively. Therefore it is thought that PDI could have two parallel optical systems, one for observing at $10 \mu$ and the other for $5 \mu$, but using the same delay lines.

For searches at both wavelength regions PDI is a system more sensitive than any other before it. It explores with an angular resolution that is higher even than planned for NGST for the same wavelength region, and is intended to observe sources as faint as 500nJy. There is considerable opportunity for serendipitous discoveries. For example, do systems with large Kuiper belt emission such as $\alpha$ PsA have giant comets, recognizable by their highly eccentric orbits? Do stars in the Hyades and Pleiades have hot giant planets orbiting them? Are there system with highly structured zodiacal dust? Are 
terrestrial planets present around perhaps half of all single stars as some suppose, or are they much rarer? Where is the nearest terrestrial type planet? And how would we optimize TPF to learn as much as possible about it? In fact we had already noticed that it is hard to optimize TPF for a mission about which so much is unknown. With PDI we do not only reduce the technological uncertainties of TPF, but we reduce the astronomical uncertainties for its design

We have not yet, at the time of writing, yet gone through the detailed analysis necessary before recommending that a precursor such as PDI should be flown. It is clearly related to TPF, but also focuses on more general topics of the nature and structure of planetary systems that have been downplayed as TPF goals. However, the arguments presented here make such an interferometer very appealing. Our near-future task is to explore the developments that would be needed to make such a mission as technically convincing as it is scientifically interesting. The prospect of using a precursor to bring in Terrestial Planet Finder faster, cheaper and better will be pursued.

\section{CONCLUSION}

A precursor to TPF appears to be technologically substantially easier than TPF, and within the price range of Discovery missions. It could take advantage of much technology that has already been developed. The science available to such a mission appears to be very interesting in the Origins framework. And the data from such a mission would be very helpful for planning TPF.

\section{ACKNOWLEDGEMENTS}

Support for TPF and SIM studies at the University of Arizona has been provided by JPL under contract \# 961268.

\section{REFERENCES}

1. C.A. Beichman A Roadmap for the Exploration of Neighboring Planetary Systems (Ex-NPS) Jet Prop. Lab. Publ. 96-22 Pasadena CA 1996

2. J.R.P. Angel J.R.P The Next Generation Space Telescope" ed. P.Y. Bely, C.J. Burrows and G.D. Illingworth p81 Space Telescope Science Institute, Baltimore MD 1990

3. A. Leger, M. Pirre and F.J. Marceau Astronomy and Astrophysics 277,309 1993

4. J.R.P Angel and N.J. Woolf Astrophysical Journal 475, 3731997

5. D. E. Backman Exozodiacal Dust Workshop NASA Ames Mountain View CA 1998 (In Press)

6. N.J. Woolf and J.R.P. Angel Adaptive Optics Optical Society of America Technical Digest series 23, 441995

7. J. Burge NGST paper at this Kona meeting

8. P.Y. Bely paper at this Kona meeting

9. P.Hinz poster paper at this Kona meeting

10. N.J. Woolf and J.R.P. Angel Planets Beyond the Solar System and the Next Generation of Space Telescopes ed. D. Soderblom ASP Conference Series 119,135 Astronomical Society of Pacific Press San Francisco CA 1997

11. J.R.P. Angel and N.J. Woolf Science with the NGST ed. E.P. Smith and A. Koreans ASP Conference Series 133,207 Astronomical Society of Pacific Press San Francisco CA 1998 